\title{
Erratum to: Grain size and geochemistry of surface sediments in northwestern continental shelf of the South China Sea
}

\author{
Guanqiang Cai $\cdot$ Li Miao $\cdot$ Hongjun Chen $\cdot$ \\ Guihua Sun $\cdot$ Jiaoqi Wu $\cdot$ Yonghang Xu
}

Published online: 24 January 2013

(C) Springer-Verlag Berlin Heidelberg 2013

\section{Erratum to: Environ Earth Sci \\ DOI 10.1007/s12665-012-2133-x}

The original article has been available online with an important omission in Acknowledgments. The complete Acknowledgments is given below, and new words add to Acknowledgments is displayed in bold.

Acknowledgments The authors thank the captain and crew of R/V "Haiyang Si Hao" (Guangzhou Marine Geological Survey) for their efforts during sample collections. We are grateful to Xing Zhang, Xuehua Zhang, and Yanmei Wang for their elegant analytical works in the lab. We especially thank Thomas Luedman (University of Hamburg) for his help in checking the English. This research was supported by the project of China Geological Survey (1212010611302), the National Natural Science Foundation of China (41006025 and 40906047), the Open fund of the Key Laboratory of Marine Geology and Environment, Chinese Academy of Sciences (MGE-2010KG01), and the Key Laboratory of Marine Hydrocarbon Resources and Environmental Geology, Ministry of Land and Resources (MRE201003). The authors also thank the two reviewers and chief editor James W. LaMoreaux for the improvement of the paper.

The online version of the original article can be found under doi:10.1007/s12665-012-2133-x.

G. Cai $\cdot$ H. Chen $\cdot$ G. Sun $\cdot$ J. Wu

Guangzhou Marine Geological Survey,

Guangzhou 510760, People's Republic of China

Present Address:

L. Miao $(\square)$

South China Sea Institute of Oceanology, Chinese Academy

of Sciences, 164 Xingangxi Road, Guangzhou,

GD 510301, People's Republic of China

e-mail: miaoli@scsio.ac.cn

Y. Xu

Third Institute of Oceanography State Oceanic Administration,

178 Daxue Road, Xiamen 361005, People's Republic of China 